to e Declaração de Nascido Vivo. Principais causas de óbito: 38\% malformações congênitas (29\% cardíacas); $21 \%$ causas infecciosas (55,4\% por sepse precoce); $11,5 \%$ asfixia perinatal; $20 \%$ causa não identificada por falta de dados. Óbitos pós-alta hospitalar: Apgar de $5^{\circ}$ minuto > sete (100\%); 61\% óbito entre 15 e 27 dv; $42 \%$ chegaram sem vida ao hospital e $16 \%$ faleceram em menos de 24 horas; $47 \%$ causa indeterminada; $33 \%$ causas infecciosas. NM: $50 \%$ causa indeterminada. Os CMPI constituem-se num importante instrumento de gestão onde é possível, após análise dos óbitos, planejar medidas de intervenção para reduzir a morte de crianças.

\title{
Sistema de Investigação dos óbitos Perinatais e Neonatais por meio de Comitês de Mortalidade Perinatal $e$ Infantil na Cidade de São Paulo
}

Geny M. Yao, Cléa R. Leone, Lílian Sadeck, Eneida R. Vico, Mauro Taniguchi

Prefeitura do Município de São Paulo. Secretaria Municipal de Saúde. Saúde da Criança e do Adolescente.

Endereço: Rua General Jardim, 36, $5^{\circ}$ andar, República, CEP 01223-010, São Paulo, SP, Brasil.

E-mail: criancadolescente@prefeitura.sp.gov.br

O objetivo deste trabalho é divulgar o fluxo da investigação do óbito infantil pelos Comitês de Mortalidade Perinatal e Infantil (CMPI) na cidade de São Paulo e apresentar os resultados de 2006. A AT analisou 412 casos, sendo 354 óbitos neonatais (ONN) e 58 natimortos (NM). Quanto aos resultados, os dados maternos apontam: idade entre 20 e 34 anos (64\%); escolaridade $\geq$ oito anos (63\%); $56 \%$ com sete ou mais consultas de pré-natal. Dados da criança: dos ONN, 24\% menores de um dia de vida (dv); $10 \%$ pós-alta hospitalar; $53 \%$ com discordância do quesito raça/cor na Declaração de Óbi- 